\title{
AN ISOPERIMETRIC INEQUALITY RELATED TO THUE'S EQUATION
}

\author{
MICHAEL A. BEAN
}

\begin{abstract}
This paper announces the discovery of an isoperimetric inequality for the area of plane regions defined by binary forms. This result has been applied subsequently in the enumeration of solutions to the Thue inequality and, given its fundamental nature, may find application in other areas as well.
\end{abstract}

\section{INTRODUCTION}

Let $F(X, Y)=a_{0} X^{n}+a_{1} X^{n-1} Y+\cdots+a_{n} Y^{n}$ be a binary form with rational integer coefficients, and let $A_{F}$ denote the area of the region $|F(x, y)| \leq 1$ in the real affine plane. In a seminal paper of 1909, Thue [11] showed that if $F$ is an irreducible form with degree at least three and if $h$ is a non-zero integer, then the equation $F(x, y)=h$ has only a finite number of solutions in integers $x$ and $y$. In 1934, Mahler [7] gave an estimate for the number, $N_{F}(h)$, of solutions of the Thue inequality $|F(x, y)| \leq h$ in terms of the area, $A_{F} h^{2 / n}$, of this region; in particular, he showed, under the same conditions as Thue, that

$$
\left|N_{F}(h)-A_{F} h^{2 / n}\right| \leq c h^{1 /(n-1)}
$$

where $c$ is a number which depends only on $F$. Mahler left $c$ and $A_{F}$ unspecified, but did show that $A_{F}$ is finite (under the above conditions).

Surprisingly little attention has been given to the estimation of $A_{F}$, even though it is clear that sharp estimates for $A_{F}$ would have significant implications for Thue inequalities. Mueller and Schmidt $[8,9]$ showed, under certain technical conditions, that $A_{F}$ is bounded when the number of coefficients of $F$ is small, but their approach gave little insight into the nature of $A_{F}$ in general. The purpose of this paper is to announce the discovery of an isoperimetric inequality for $A_{F}$. Detailed proofs will be forthcoming in [4].

Theorem 1. Let $F$ be a binary form with integer coefficients having non-zero discriminant and degree at least three. Then

$$
A_{F} \leq 3 B\left(\frac{1}{3}, \frac{1}{3}\right)
$$

where $B\left(\frac{1}{3}, \frac{1}{3}\right)$ denotes the Beta function with arguments of $\frac{1}{3}$. This bound is attained for forms with integer coefficients which are equivalent under $\mathrm{GL}_{2}(\mathbb{Z})$ to $X Y(X-Y)$.

The approximate numerical value of $3 B\left(\frac{1}{3}, \frac{1}{3}\right)$ is 15.8997 . A similar result is true for forms with complex coefficients provided that one includes a factor

Received by the editors August 27, 1993.

1991 Mathematics Subject Classification. Primary 11D75, 11J25, 51M25; Secondary 26D20, 33B15.

Supported in part by a Postdoctoral Fellowship from the Natural Sciences and Engineering Research Council of Canada. 


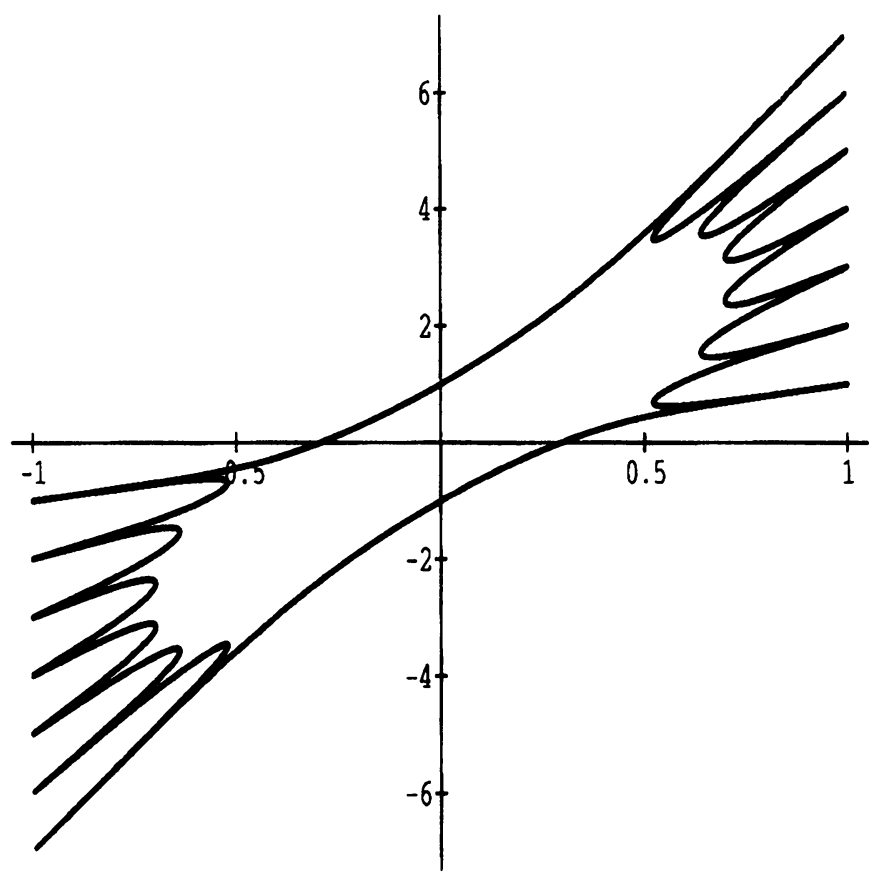

Figure 1. $\left|P_{7}(x, y)\right|=1$

involving the discriminant of $F$ (see $\S 2$ ). However, the conditions on the discriminant and the degree cannot be relaxed in general since, for example, the forms $X^{n}$ and $X^{2}-Y^{2}$ give rise to infinite area. The condition that $F$ have non-zero discriminant is equivalent to the condition that neither $F(x, 1)$ nor $F(1, y)$ have multiple roots and is satisfied, in particular, when $F$ is an irreducible form. The notion of equivalence under $\mathrm{GL}_{2}(\mathbb{Z})$ will be explained in $\S 2$.

One of the striking aspects of Theorem 1 is that the bound given for $A_{F}$ is relatively small. In light of Mahler's result, one might expect $N_{F}(1)$ to be bounded and small as well. However, an examination of the forms

$$
P_{k}(X, Y)=X^{2 k}+(X-Y)^{2}(2 X-Y)^{2} \cdots(k X-Y)^{2}
$$

reveals that this is not the case. Indeed, the region $\left|P_{k}(x, y)\right| \leq 1$ contains at least $2 k$ integer lattice points, namely,

$$
\pm(1,1), \pm(1,2), \ldots, \pm(1, k),
$$

but nevertheless is bounded (since $P_{k}$ is positive definite) and has area less than 16. (See Figure 1 for the graph of $P_{7}$.)

These examples suggest that a finer analysis of the error term in Mahler's result could be quite delicate. However, they raise the interesting question of classifying forms according to the size of this error.

\section{THE MAIN IDEAS}

There are three main ideas involved in the proof of Theorem 1. The first and most important is to consider the estimation of $A_{F}$ over the larger class of forms having complex coefficients. 
For any form $F$, let $D_{F}$ denote the discriminant of $F$. If $F$ has a factorization $\prod_{i=1}^{n}\left(\alpha_{i} X-\beta_{i} Y\right)$ with $\alpha_{i}, \beta_{i} \in \mathbb{C}$ (every binary form with complex coefficients has such a factorization), then $D_{F}=\prod_{i<j}\left(\alpha_{i} \beta_{j}-\alpha_{j} \beta_{i}\right)^{2}$. Let $\mathrm{GL}_{2}(\mathbb{R})$ denote the group of $2 \times 2$ real invertible matrices. For each $T=\left(\begin{array}{ll}a & b \\ c & d\end{array}\right) \in \mathrm{GL}_{2}(\mathbb{R})$, let $F_{T}(X, Y)=F(a X+b Y, c X+d Y)$. Two forms are said to be equivalent under $\mathrm{GL}_{2}(\mathbb{R})$ if $G=F_{T}$ for some $T \in \mathrm{GL}_{2}(\mathbb{R})$. Equivalence under $\mathrm{GL}_{2}(\mathbb{Z})$ is defined similarly.

In this more general setting, we can prove the following inequality.

Theorem 2. Let $F$ be a binary form with complex coefficients having degree $n \geq 3$ and discriminant $D_{F} \neq 0$. Then

$$
\left|D_{F}\right|^{1 / n(n-1)} A_{F} \leq 3 B\left(\frac{1}{3}, \frac{1}{3}\right) \text {. }
$$

This bound is attained precisely when $F$ is a cubic form which, up to multiplication by a complex number, is equivalent under $\mathrm{GL}_{2}(\mathbb{R})$ to the form $X Y(X-Y)$.

Since the discriminant of a form with integer coefficients is an integer, Theorem 1 is an immediate consequence. The inequality in Theorem 2 is an isoperimetric inequality since it is sharp and connects the geometric quantities $A_{F}$ and $\left|D_{F}\right|$ which are related to the shape and size of the region $|F(x, y)| \leq 1$. The quantity $\left|D_{F}\right|^{1 / n(n-1)} A_{F}$ is natural to consider since it is invariant under $\mathrm{GL}_{2}(\mathbb{R})$ (i.e., $\left|D_{F_{T}}\right|^{1 / n(n-1)} A_{F_{T}}=\left|D_{F}\right|^{1 / n(n-1)} A_{F}$ for all $\left.T \in \mathrm{GL}_{2}(\mathbb{R})\right)$ in the same way that $A_{F}$ is invariant under $\mathrm{GL}_{2}(\mathbb{Z})$.

The real advantage of considering $\left|D_{F}\right|^{1 / n(n-1)} A_{F}$ instead of $A_{F}$ is that one can apply the inequality between arithmetic and geometric means in a systematic fashion to reduce the estimation of $\left|D_{F}\right|^{1 / n(n-1)} A_{F}$ to cubic forms. This is the second main idea. To be specific, let

$$
M_{n}=\max \left|D_{F}\right|^{1 / n(n-1)} A_{F}
$$

where the maximum is taken over all forms of degree $n$ with $D_{F} \neq 0$. Then one can show that $\left\{M_{n}\right\}$ is a decreasing sequence.

The third main idea is to consider the quantity $\left|D_{F}\right|^{1 / n(n-1)} A_{F}$ as a function of $n$ complex variables (the roots of $F(x, 1)$ ). By appealing to an appropriate maximum principle, one can then deduce that $\left|D_{F}\right|^{1 / n(n-1)} A_{F}$ is maximized by a form $F$ for which $F(x, 1)$ has $n$ distinct real roots. Heuristically, one expects $\left|D_{F}\right|^{1 / n(n-1)} A_{F}$ to be maximized by a form whose corresponding graph $|F(x, y)|=1$ has the maximal number of asymptotes in the real affine plane (i.e. by a form for which $F(x, 1)$ has $n$ distinct real roots). In the case of cubics, every such form is equivalent under $\mathrm{GL}_{2}(\mathbb{R})$ to $X Y(X-Y)$.

\section{Related Questions}

The outline given above raises some interesting questions regarding the sequence $\left\{M_{n}\right\}$. In particular, one might like to know all the values of this sequence and its limiting behavior. These and other questions will be addressed in [3]. However, based on that work, we believe that the following is true.

Conjecture. The maximal value $M_{n}$ of the quantity $\left|D_{F}\right|^{1 / n(n-1)} A_{F}$ over the class of forms of degree $n$ with complex coefficients and non-zero discriminant 
is attained precisely when $F$ is a form which, up to multiplication by a complex number, is equivalent under $\mathrm{GL}_{2}(\mathbb{R})$ to the form

$$
F_{n}^{*}(X, Y)=\prod_{k=1}^{n}\left(X \sin \left(\frac{k \pi}{n}\right)-Y \cos \left(\frac{k \pi}{n}\right)\right) .
$$

Moreover, the sequence of maximal values $\left\{M_{n}\right\}$ decreases monotonically to the value $2 \pi$.

\section{ACKNOWLEDGMENT}

The author is grateful to E. Bombieri, J. Friedlander, C. Stewart, and the referee for their suggestions and comments.

\section{REFERENCES}

1. M. Abramowitz and I. Stegun, Handbook of mathematical functions, Dover, New York, 1965.

2. M. A. Bean, Areas of plane regions defined by binary forms, Ph.D. thesis, Univ. of Waterloo, 1992.

3. _ Binary forms, hypergeometric functions, and the Schwarz-Christoffel mapping formula, submitted.

4. $\_$, An isoperimetric inequality for the area of plane regions defined by binary forms, Compositio Math. (to appear).

5. M. A. Bean and J. L. Thunder, Isoperimetric inequalities for volumes associated with decomposable forms, submitted.

6. G. Hardy, J. E. Littlewood, and G. Polya, Inequalities, Cambridge Univ. Press, Cambridge, 1952.

7. K. Mahler, Zur Approximation algebraischer Zahlen III, Acta Math. 62 (1934), 91-166.

8. J. Mueller and W. M. Schmidt, On the Newton polygon, Monatsh. Math. 113 (1992), 33-50.

9. __ Thue's equation and a conjecture of Siegel, Acta Math. 160 (1988), 207-247.

10. W. M. Schmidt, Diophantine approximations and diophantine equations, Lecture Notes in Math., vol. 1467, Springer-Verlag, New York, 1991.

11. A. Thue, Über Annäherungswerte algebraischer Zahlen, J. Reine Angew. Math. 135 (1909), 284-305.

12. J. L. Thunder, The number of solutions to cubic Thue inequalities, Acta Arith. (to appear).

Mathematical Sciences Research Institute, 1000 Centennial Drive, Berkeley, CaliFORNIA 94720

Current address: Department of Mathematics, University of Toronto, Toronto, Ontario M5S 1A1, Canada

E-mail address: bean@math.toronto.edu 